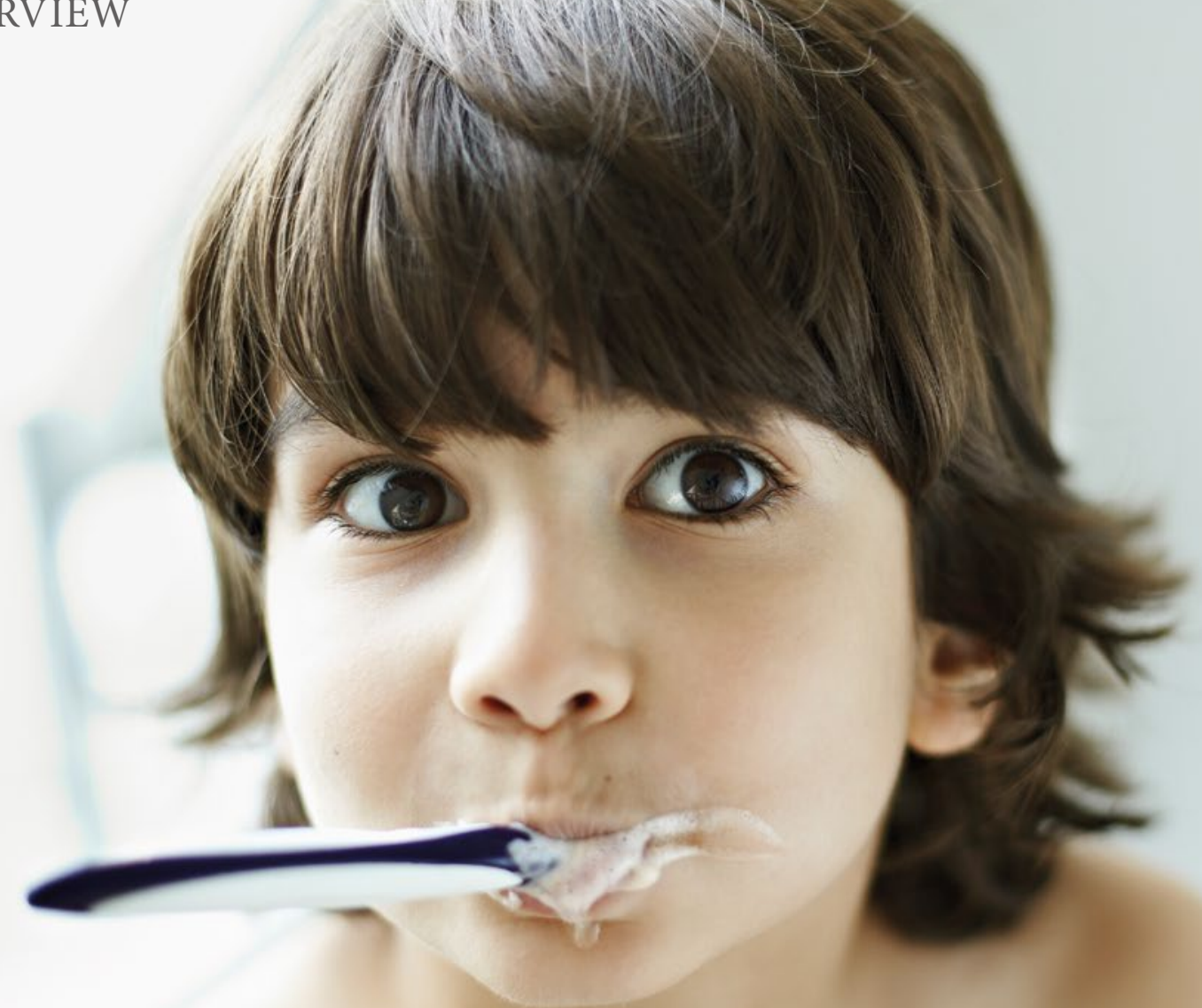

\title{
The future of Dental Public Health
}

\section{David Westgarth}

Editor, BDJ in Practice

I t's easy to forget dental decay is almost entirely preventable. And, at a time when public health messages are of the utmost importance, you wonder how much ground the profession has lost in 2020 trying to get that message across to patients.

Following the announcement of Public Health England's closure, should dentistry and dental public health in particular be concerned about the direction of travel? I asked Rob Witton, Chair of the BDA Dental Public Health Committee, about what the future holds.

Before we talk about COVID-19, what did the future of dental public health look like pre-pandemic?

RW Dental public health has always been a small speciality, but in my opinion one that always punches above its weight. The last re-organisation of dental public health placed the majority of the workforce in Public Health England either working in a national team or in regions across England. In addition to this, we also have academic colleagues who play a crucial role in developing the evidence-base for the speciality, supporting education and training and identifying research gaps and priorities. Before the pandemic, this eco-system was working successfully, and dental public health teams have made a huge contribution to progressing the dental agenda at national and regional level. I know in my own conversations with partners and stakeholders that dental public health input is highly valued and sought after. However, in some areas, particularly in remote and rural parts of the country, there have been persistent challenges in recruiting to vacant positions and this is an ongoing concern. The good news for dental public health is that we have an excellent group of trainees moving through the system and this healthy supply of enthusiasm and talent bodes well for the future of dentistry and the speciality.

\section{Workforce was an issue. What concerns do you have, and have they been exacerbated by the pandemic?}

RW Workforce issues are an ongoing concern and COVID-19 has further highlighted how thinly spread we are as a specialty across the country. Despite this, the dental public health response to COVID-19 has been remarkable, mirroring that seen across the dental community. I have seen at first hand the hard work and dedication of my dental public health colleagues in supporting dentistry and the wider NHS. This is often not headline grabbing or immediately visible to dental teams but nonetheless it is there in the background influencing, enabling and facilitating. Wherever we end up as a speciality, we need to ensure the value of dental public health is recognised and promoted. 
The news that PHE was to be scrapped took many of us by surprise. Why is it important to dental health in England?

RW The need for dental public health advice is now more important than ever as we recover from the pandemic and restore dental services and oral health improvement programmes. As PHE is the home for the majority of specialists or consultants in dental public health, it is crucial the workforce is secured in any re-organisation of Public Health England. Dental public health provides independent advice and support to organisations across the entire health and social care system and this must be preserved to enable us to ensure patient needs are met and heard, and system partners prioritise population oral health. If we as a dental community are serious about improving the oral health of the population and reducing oral health inequalities dental public health leadership and expertise is a vital part of the mix.

\section{From what you know, how confident - or otherwise - are you that its} replacement, whatever that is, will be of benefit to dental public health?

RW Right now, this is a difficult question to answer, as we have not seen any proposals on the future of the dental public health workforce and where it will sit within the new system. I hope more information will become available in the New Year so we as a professional group, and with support from the BDA and BASCD can determine how we respond to any proposals through the consultation.

\section{The FDI Vision 2030 paper aims to 'support the integration of oral health into the wider context of health and sustainability'. Is that really possible?}

RW Yes, it is possible, but we need to influence the wider agendas in health and social care to see oral health as an important part of patients' overall NHS care. At the moment oral health and dentistry are the Cinderella services with little prioritisation and value in the wider NHS. The links between oral health and general health are well documented and obvious to us in the dental community but we still have some way to go in fully integrating oral care into whole patient care. Being able to influence the wider social determinants of health is also an important role for dental public health and one that recognises the impact that adverse socio-economic and environmental circumstances can have on poor oral health outcomes. Linked to this is climate change, and dentistry will need to consider how it can be more sustainable to support the NHS in its ambition to be carbon neutral by 2040 .

\section{'Before the pandemic, this eco-system was working successfully, and dental public health teams have made a huge contribution to progressing the dental agenda at national and regional level.'}

Is the money really there to support the need to integrate oral health into wider public health messages?

RW If I am honest it isn't, and oral health really needs increased investment to see tangible improvement. This has to be routed through local authorities (where statutory responsibilities for health improvement rests) to enable them to develop programmes with increased reach, engagement and impact in communities, and through NHS England and Improvement to enable dental teams to deliver effective prevention and health promotion for their patients as part of their contract. Both need funding properly.

The BDA along with 55 other national organisations recently wrote to HM Treasury on the matter of public health funding and the need to prioritise and adequately fund disease prevention and health improvement functions locally, regionally and nationally. The BDA highlighted in the response the importance of community water fluoridation and oral health improvement programmes such as supervised tooth brushing. It is a message we have to raise at the highest level and through every means possible.

\section{What one thing absolutely needs to happen this year to protect dental public health?}

RW I hope in the future specialists and consultants in dental public health will have a stable and secure base to work from, and the dental public health workforce will not be required to sing for its supper in every NHS re-organisation of the future. As a professional group of dentists, we are, and have been, subject to significant disruption every time the NHS re-structures, and we all recognise that while deck chairs are shuffled around normal day-to-day work on tackling the issues in dentistry suffers. We are the only dental speciality to be subject to such regular upheaval in this way.

I really want to see a longer-term solution for my colleagues that enables us to build a sustainable speciality, and one that empowers us to use the full range of our skills and expertise for the benefit of patients and population health working across the three pillars of public health - health improvement, healthcare public health and health protection. The first step on this journey is having the full support of the dental community behind us to achieve this.

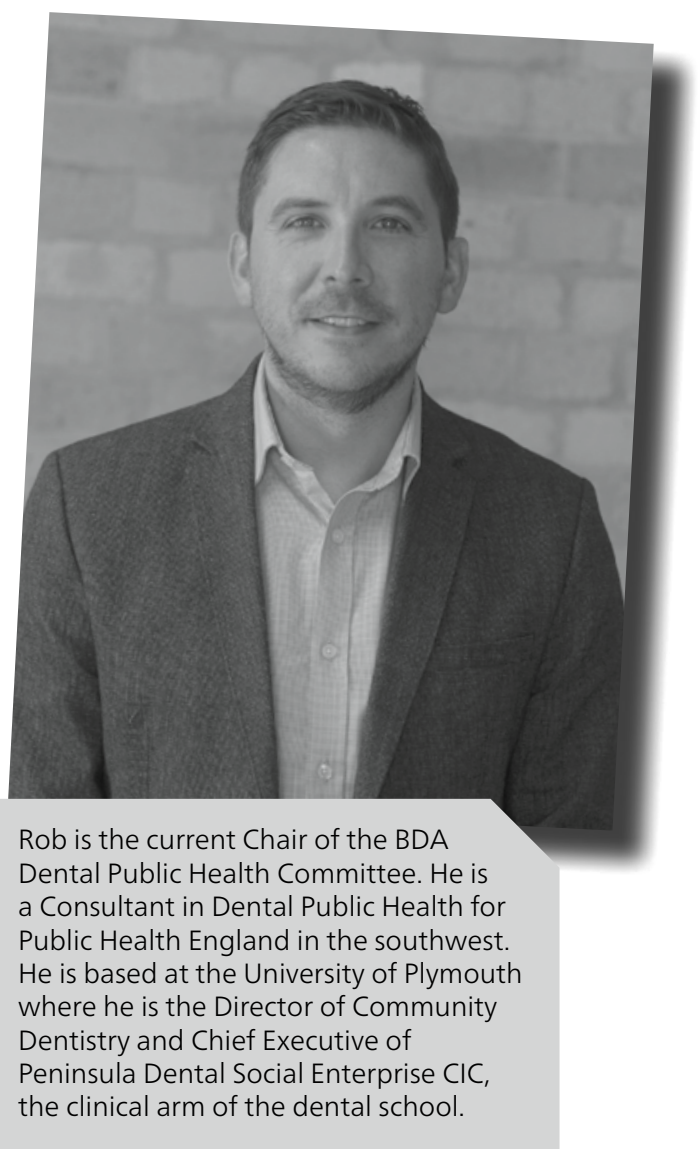

https://doi.org/10.1038/s41404-020-0621-3 